\title{
LONGITUDINALLY EXTENSIVE TRANSVERSE MYELITIS DUE TO NEUROTUBERCULOSIS IN PATIENT WITH SYSTEMIC LUPUS ERYTHEMATOSUS: A CASE REPORT
}

\author{
Jessica Carvalho da Silva ${ }^{1, \star}$, Thayana Evelling Uchoa da Silva ${ }^{1}$, Bárbara Seabra Carneiro ${ }^{1}$, Rosana Barros de Souza Carneiro ${ }^{1}$, \\ Luiz Fernando de Souza Passos ${ }^{1}$ \\ 1.Universidade Federal do Amazonas, Manaus (AM), Brazil. \\ ${ }^{\star}$ Corresponding author: jessicacarvalho1202@gmail.com
}

\section{BACKGROUND}

Longitudinally extensive transverse myelitis (LETM) is conceptually the presence of inflammatory lesions in three or more contiguous spinal cord segments. The etiologies that course with LETM include collagenosis, neuromyelitis optica (NMO), infection, acute disseminated encephalomyelitis and others. Tuberculosis (TB) transverse myelitis is very rare, which makes TB myelopathy associated with a longitudinally extensive lesion a much rarer clinical entity. We report a case of LETM, with negative anti-aquaporin 4 (AQP4) antibody without optic neuritis in a young woman with consequent diagnosis of neurotuberculosis.

\section{CASE REPORT}

A 23-year-old woman presented with sudden onset of intense neuropathic pain in lower limbs, which quickly progresses to paraplegia and anesthesia at level T12, associated with urinary retention and fecal incontinence. Event preceded by mild flulike symptoms, lasting 2 weeks, ruled out arboviruses and coronavirus infection. The patient's history included systemic lupus erythematosus (SLE), under irregular treatment regimens. Investigation performed with immediate implementation of spinal cord MRI, examination of cerebrospinal fluid (CSF), AQP4 testing and infection screening. Gadolinium contrast MRI revealed a longitudinal extensive spinal cord lesion from level $\mathrm{C} 2$ to the medullary cone, with local expansive effect, but without indication of conduct by neurosurgery. The CSF analysis showed mild elevation of cell count and protein levels, low glucose levels and no oligoclonal bands. Due to a history of irregular treatment and a sudden clinical onset associated with extensive spinal cord injury, treatment of LETM caused by SLE was chosen. Pulse therapy with methylprednisolone was performed in association with plasmapheresis. After that was received the result of Mycobacterium tuberculosis polymerase chain reaction on the CSF, which came out positive, immediately started treatment for the infectious disease. Despite the treatment for neurotuberculosis started only 5 days after initial deficit, patient maintains paraplegia, because of that opted for therapy with human immunoglobulin. After 1 month of clinical follow-up the same neurological deficit remains.

\section{CONCLUSION}

Longitudinally extensive transverse myelitis is a characteristic feature of neuromyelitis optica, but such spinal lesions can also occur in various other autoimmune and inflammatory diseases or in infectious diseases with CNS involvement. This case report is able to exemplify the difficulty in differential diagnosis between infectious and autoimmune causes. Longitudinally extensive transverse myelitis caused by TB is a rare entity, with few reports in the literature; however, extrapulmonary tuberculosis is a common impairment in endemic areas. Due to the high morbidity caused by the disease, initiation of targeted therapy may result in better long-term outcome.

\section{KEYWORDS}

Transverse neuromyelitis, Neurotuberculosis, Systemic lupus erythematosus. 